\title{
Inhibition of plasminogen activator inhibitor-1 attenuates against intestinal fibrosis in mice
}

\author{
Jin Imai ${ }^{1,2}$, Takashi Yahata ${ }^{3,4}$, Hitoshi Ichikawa ${ }^{2}$, Abd Aziz Ibrahim ${ }^{3,5}$, Masaki Yazawa ${ }^{6}$, Hideaki Sumiyoshi ${ }^{1,7}$, \\ Yutaka Inagaki ${ }^{1,7}$, Masashi Matsushima ${ }^{2}$, Takayoshi Suzuki ${ }^{2}$, Tetsuya Mine ${ }^{2}$, Kiyoshi Ando ${ }^{3,5}$, Toshio Miyata ${ }^{8}$, \\ Katsuto Hozumi ${ }^{1,3,6}$ \\ ${ }^{I}$ Center for Matrix Biology and Medicine, ${ }^{2}$ Department of Gastroenterology, and ${ }^{3}$ Research Center for Regenerative Medicine, Tokai University \\ School of Medicine, Kanagawa; Departments of ${ }^{4}$ Cell Transplantation and Regenerative Medicine, ${ }^{5}$ Hematology and Oncology, ${ }^{6}$ Immunology, \\ and ${ }^{7}$ Regenerative Medicine, Tokai University School of Medicine, Kanagawa, ${ }^{8}$ Division of Molecular Medicine and Therapy, Tohoku University \\ Graduate School of Medicine, Sendai, Japan
}

Background/Aims: Intestinal fibrosis is a major complication of Crohn's disease (CD). The profibrotic protein transforming growth factor- $\beta$ (TGF- $\beta$ ) has been considered to be critical for the induction of the fibrotic program. TGF- $\beta$ has the ability to induce not only the expression of extracellular matrix (ECM) including collagen, but also the production of plasminogen activator inhibitor-1 (PAI-1) that prevents enzymatic degradation of the ECM during the onset of fibrotic diseases. However, the significance of PAI-1 in the developing intestinal fibrosis has not been fully understood. In the present study, we examined the actual expression of PAI-1 in fibrotic legion of intestinal inflammation and its correlation with the abnormal ECM deposition. Methods: Chronic intestinal inflammation was induced in BALB/c mice using 8 repeated intrarectal injections of 2,4,6-trinitrobenzene sulfonic acid (TNBS). TM5275, a PAI-1 inhibitor, was orally administered as a carboxymethyl cellulose suspension each day for 2 weeks after the sixth TNBS injection. Results: Using a publicly available dataset (accession number, GSE75214) and TNBS-treated mice, we observed increases in PAI-1 transcripts at active fibrotic lesions in both patients with CD and mice with chronic intestinal inflammation. Oral administration of TM5275 immediately after the onset of intestinal fibrosis upregulated MMP-9 (matrix metalloproteinase 9) and decreased collagen accumulation, resulting in attenuation of the fibrogenesis in TNBS-treated mice. Conclusions: PAI-1-mediated fibrinolytic system facilitates collagen degradation suppression. Hence, PAI1 inhibitor could be applied as an anti-fibrotic drug in CD treatment. (Intest Res 2020;18:219-228)

Key Words: Plasminogen activator inhibitor-1; Matrix metalloproteinase 9; Intestinal fibrosis; Crohn disease; 2,4,6-Trinitrobenzene sulfonic acid

\section{INTRODUCTION}

Crohn's disease (CD) is a chronic inflammatory condition of diverse etiology. Chronic inflammation lead to serious complications, especially extensive local intestinal fibrosis and digestive obstruction. Approximately $40 \%$ of CD patients devel-

Received March 29, 2019. Revised October 2, 2019.

Accepted October 7, 2019.

Correspondence to Katsuto Hozumi, Department of Immunology, Tokai

University School of Medicine, 143 Shimokasuya Isehara, Kanagawa 259-

1193, Japan. Tel: +81-463931121, Fax:+81-463942976, E-mail: hozumi@

is.icc.u-tokai.ac.jp op clinically apparent strictures. ${ }^{1}$

According to the different therapeutic approaches used in clinical practice, in addition to mixed types, the strictures can be subdivided into fibrotic and inflammatory types. Standard anti-inflammatory therapies, including treatment with antiTNF- $\alpha$ antibodies, can improve inflammatory strictures by minimizing inflammation-mediated edema. Anti-inflammatory therapies currently used for IBD neither prevent nor reverse established fibrotic strictures, which may present years after the remission of active inflammation. ${ }^{2}$ The immunological mechanisms related to acute phase of intestinal inflamma- 
tion and repair have been extensively studied; in contrast, the pathophysiology of chronic mucosal wound healing and the late events of repair leading to fibrosis remain largely unexplored. ${ }^{3}$ Therefore, an understanding of the cellular and molecular mechanisms of intestinal fibrosis and the development of a specific anti-fibrotic therapy for stricture complications in patients with $\mathrm{CD}$ are required because the existing therapeutic approaches are inadequate for addressing the problem. ${ }^{4}$

In tissue fibrosis, accumulation of collagen occurs due to an imbalance between enhanced deposition and reduced degradation of the extracellular matrix (ECM) comprising mainly collagen. ${ }^{5}$ Several reports have demonstrated that the exaggerated tissue deposition of collagen during the fibrotic process is largely due to an increase in the transcription of COL1A1 and COL1A2, which is governed by TGF- $\beta$ and its intracellular mediators Smad proteins. ${ }^{6}$ We previously reported that interferon- $\gamma$ and its downstream effector Y-box binding protein-1 inhibit COL1A2 transcription ${ }^{7}$ and that HSc025, a small compound, promotes nuclear Y-box binding protein-1 translocation, thereby resulting in reduced TGF- $\beta$ production. To investigate the significance of transforming growth factor- $\beta$ (TGF- $\beta$ ) and its signal transduction for the intestinal fibrosis, we took the chronic colitis model in mice induced by the frequent administrations of 2,4,6-trinitrobenzene sulfonic acid (TNBS), because the intestinal fibrosis has never been observed in the acute colitis model. Using this model, we showed that oral administration of HSc025 decreases TNBS-induced fibrosis in the gut, as well as in other organs, by reducing TGF- $\beta$ production, suggesting the critical role of TGF- $\beta$ for the intestinal fibrogenesis. $^{7,8}$

Of note, plasminogen activator inhibitor-1 (PAI-1) is another major downstream target of TGF- $\beta$ signal transduction. The transcription of PAI- 1 is directly regulated by Smad $3 .{ }^{9}$ PAI- 1 levels are reportedly elevated in fibrotic tissues; this disturbs the proteolytic activities of matrix metalloproteinases (MMPs) via tissue plasminogen activator (tPA) and plasmin, resulting in decreased rates of collagen degradation and tissue fibrogenesis. ${ }^{10}$ Very recently, it has been reported that PAI-1 expression was highly enriched in active lesions in both patients with IBD and mice with experimental colitis, and confirmed that PAI- 1 and its direct target, tPA, played an important role in regulating intestinal inflammation. ${ }^{11}$ However, their functions have been only validated in the acute phase of experimetal colitis and its correlation with intestinal fibrogenesis observed in the chronic phase has remained obscure.

In the present study, we confirmed the increase in PAI- 1 transcripts at the active fibrotic lesions in the terminal ileum of patients with $\mathrm{CD}$, which was closely correlated with those of TGF- $\beta 1$ and COL1A2. These correlations were also observed in TNBS-treated mice with intestinal fibrosis, and the oral administration of TM5275, originally developed PAI-1 inhibitor, ameliorates the pathogenesis in the chronic colitis model. Such observations provide novel insights into the potential treatment strategies for intestinal fibrosis in patients with CD.

\section{METHODS}

\section{Mice and Induction of Chronic Colitis}

Female (6-week-old) BALB/c mice were from CLEA Japan Inc. (Tokyo, Japan). Mice were maintained in a specific pathogen-free environment. All experiments were approved by the Animal Experimentation Committee of Tokai University, Kanagawa, Japan (approved Nos. \#183031 and \#194027). Fibrotic inflammation was produced via intrarectal injection by $3.5-\mathrm{F}$ catheter instillation of a $2 \%$ solution of TNBS (Research Organics, Cleveland, OH, USA) in 50\% ethanol under light anesthesia with isoflurane. TNBS was provided as 8 weekly injections, with the dose sequentially increasing in the $0.4-1.6 \mathrm{mg}$ range $(0.4,0.4,0.8,0.8,1.2,1.2,1.6$, and $1.6 \mathrm{mg})$. The mice were picked up food for 24 hours before TNBS injection and were held vertically for 60 seconds after the intrarectal injection. The ethanol group mice injected a similar volume of $50 \%$ ethanol. Tissues were harvested 3 days after the completion of the 8th times injections. The colon was opened longitudinally and snap frozen for preparation of frozen sections or RNA extraction. The formalin-fixed paraffin-embedded sections were prepared for H\&E or Masson trichrome staining as collagen detection.

\section{Administration of PAI- 1 Inhibitor}

TM5275 is a specific inhibitor of PAI-1 molecules, and does not interfere with other serpin/serine protease systems. ${ }^{12}$

TM5275 was orally administered as a carboxymethyl cellulose (CMC) suspension each day for 2 weeks after the 6th TNBS injection. The treatment had no effect on body weight or food intake throughout the experimental duration.

\section{Experimental Design}

The following groups of mice were included in the present chronic study: (1) ethanol-treated mice $(\mathrm{n}=10)$; (2) TNBS and CMC-treated mice ( $\mathrm{n}=10)$; (3) TNBS and $10 \mathrm{mg} / \mathrm{kg} / \mathrm{day}$ TM5275-treated mice ( $\mathrm{n}=9$ ); or (4) TNBS and $50 \mathrm{mg} / \mathrm{kg} / \mathrm{day}$ TM5275-treated mice $(\mathrm{n}=10)$. 


\section{Histopathological Evaluation and Image Analysis of Intestinal Fibrosis}

The evaluator blinded to the groups scored the macroscopic colonic lesions which is shown in Table $1 .^{7,13}$

Specimens obtained from the colons of all groups were immersed in $10 \%$ buffered formalin in phosphate-buffered saline overnight at room temperature, and followed by the standard procedure for paraffin embedding. H\&E stain assess the degree of inflammation and then with collagen staining to detect tissue fibrosis. The evaluator who was unaware of the group assignments, scored all the microscopic histological scores which is shown in Table $1 .^{14}$

\section{Collagen Assay Methods}

Intestinal collagen content was evaluated using the Sircol Collagen Assay Kit (Biocolor, Carrickfergus, UK). The colonic specimens obtained from of all mice were homogenized in $0.5 \mathrm{M}$ acetic acid containing $1 \%$ pepsin. The resulting mixture was stirred over night at $4^{\circ} \mathrm{C}$. The total soluble collagen content of the mixture was determined. The acid-soluble type-I collagen supplied with the kit was used to generate a standard curve. ${ }^{15}$

\section{Quantitative PCR Assay}

mRNA expressions of murine Pai-1 in the snap-frozen samples were assessed using quantitative PCR (qPCR). The amounts of target mRNA were quantified as described previously. ${ }^{16}$ Total RNA (50 ng) was reverse transcribed using ImProm-II reverse transcriptase (Promega). The primers used for amplification of Pai- 1 was 5'- GACACCCTCAGCATGTTCATC-3' (forward primer) and 5'- AGGGTTGCACTAAACATGTCAG $-3^{\prime}$ (reverse primer). The relative mRNA expressions of Pai-1 was normalized against that of the glyceraldehyde-3-phosphate dehydrogenase (Gapdh), gene in the same RNA preparation. qPCR was performed using SYBR Green qPCR Kits.

\section{Detection of MMP-9 in the Colon}

MMP-9 expression in the colon was evaluated using an ELISA kit (R\&D Systems Inc., Minneapolis, MN, USA). Frozen colon tissues were grounded in phosphate-buffered saline with $1 \%$ protease inhibitor cocktail and $1 \%$ phosphatase inhibitor cocktail. After centrifugation using the Shake Master NEO system $\left(15,000\right.$ rpm, 90 seconds, $4^{\circ} \mathrm{C}$; Biomedical Science, Kobe, Japan), the supernatants of the homogenate were collected. The protein concentrations were determined using DC protein assays (Bio-Rad, Hercules, CA, USA).
Table 1. Score of Colonic Fibrosis

\begin{tabular}{|c|c|c|}
\hline Scoring & & Score \\
\hline \multicolumn{3}{|l|}{ Macroscopic score } \\
\hline \multirow[t]{3}{*}{ Adhesions } & Absent & 0 \\
\hline & Mild/focal-zonal & 1 \\
\hline & Severe/diffuse & 2 \\
\hline \multirow[t]{3}{*}{ Thickness } & Normal & 0 \\
\hline & Mild increase & 1 \\
\hline & Severe increase & 2 \\
\hline \multirow[t]{3}{*}{ Strictures } & Absent & 0 \\
\hline & Mild & 1 \\
\hline & Severe & 2 \\
\hline \multirow[t]{3}{*}{ Dilation } & Absent & 0 \\
\hline & Mild & 1 \\
\hline & Severe & 2 \\
\hline \multirow[t]{3}{*}{ Mucosal edema } & Absent & 0 \\
\hline & Mild & 1 \\
\hline & Severe & 2 \\
\hline \multirow[t]{3}{*}{ Mucosal ulcers } & Absent & 0 \\
\hline & 1 Or 2 ulcers at one site & 1 \\
\hline & Ulcers at several sites & 2 \\
\hline Maximum possible score & & 12 \\
\hline \multicolumn{3}{|l|}{ Microscopic score } \\
\hline \multirow[t]{3}{*}{ Ulcerations } & Absent & 0 \\
\hline & Small ulcers & 1 \\
\hline & Large ulcers & 2 \\
\hline \multirow[t]{4}{*}{ Degree of inflammation } & Absent & 0 \\
\hline & Mild & 1 \\
\hline & Moderate & 2 \\
\hline & Severe & 3 \\
\hline \multirow[t]{4}{*}{ Depth of lesions } & Absent & 0 \\
\hline & Submucosa & 1 \\
\hline & Muscularis propria & 2 \\
\hline & Serosa & 3 \\
\hline \multirow[t]{3}{*}{ Degree of fibrosis } & Absent & 0 \\
\hline & Mild & 1 \\
\hline & Severe & 2 \\
\hline Maximum possible score & & 10 \\
\hline
\end{tabular}

\section{Statistical Analysis}

All analyses were performed using Graph Prism5.0 (GraphPad Software Inc. San Diego, CA, USA). Between-group differences were evaluated using the Mann-Whitney $U$-test (nonparametric). For the comparison of more than 3 groups, statis- 
tical analyses were performed using two-way ANOVA (parametric) or the Kruskal-Wallis test (nonparametric), followed by Bonferroni correction for parametric samples as a post-hoc test. $P<0.05$ was considered statistically significant.

\section{RESULTS}

\section{PAI-1 Is Overexpressed in the Terminal Ileum of Patients with CD}

An increase in TGF- $\beta$ production, which directly induces PAI1 transcription, is correlated with the abnormal deposition of collagen during the onset of fibrotic disease. ${ }^{6}$ Therefore, we validated the expression of PAI-1 in patients with CD. Intestinal fibrotic strictures are most commonly located in the terminal ileum, ${ }^{17}$ therefore, using a publicly available dataset (accession number GSE75214), ${ }^{18}$ we discovered that PAI-1 transcript was significantly upregulated in the terminal ileal mucosa of active patients with $\mathrm{CD}$ than in that of normal controls or inactive patients with CD (Fig. 1A). In addition, we confirmed the correlation between the expressions of PAI- 1 and $T G F-\beta 1$ and demonstrated that PAI-1 transcript was positively regulated by TGF- $\beta 1$ in patients with $\mathrm{CD}$ (Fig. $1 \mathrm{~B}$ ). Of note, the correlation coefficients between $P A I-1$ and $C O L 1 A 1$ and between PAI- 1 and COL1A2 were 0.73 and 0.75 , respectively (Fig. 1B). These results indicated that PAI- 1 expression increased with fibrosis-related genes in the fibrotic ileal lesion of patients with CD.

\section{Pai-1 Expression Is Also Detected in TNBS-Induced Murine Fibrotic Model}

To examine whether Pai-1 expression facilitates intestinal fibrosis, we employed a TNBS-induced murine chronic colitis model. In the model, intestinal fibrosis gradually emerges with transition from the acute inflammatory phase to the chronic phase. ${ }^{7,15}$ As demonstrated in our previous report, collagen deposition is completed following intrarectal injection of TNBS in the 8th week after the initial administration. ${ }^{7}$ TNBStreated mice exhibited obvious collagen deposition, particularly in the submucosal layer (H\&E and Masson trichrome staining) (Fig. 2A). A similar trend was observed in the collagen assay for quantifying actual deposition (Fig. 2B). We previously revealed that TGF- $\beta 1$ production is increased in the colonic mucosa of TNBS-treated mice. ${ }^{7}$ In addition, we detected an increase in local Pai-1 expression in fibrotic lesions when
A

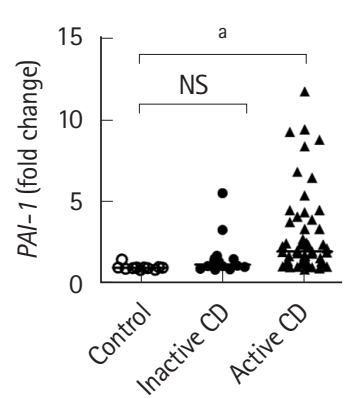

B
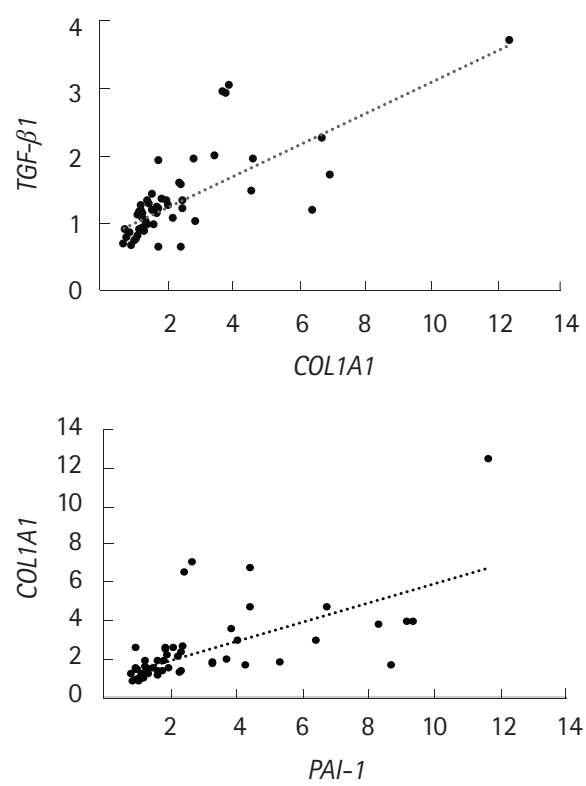

Active CD
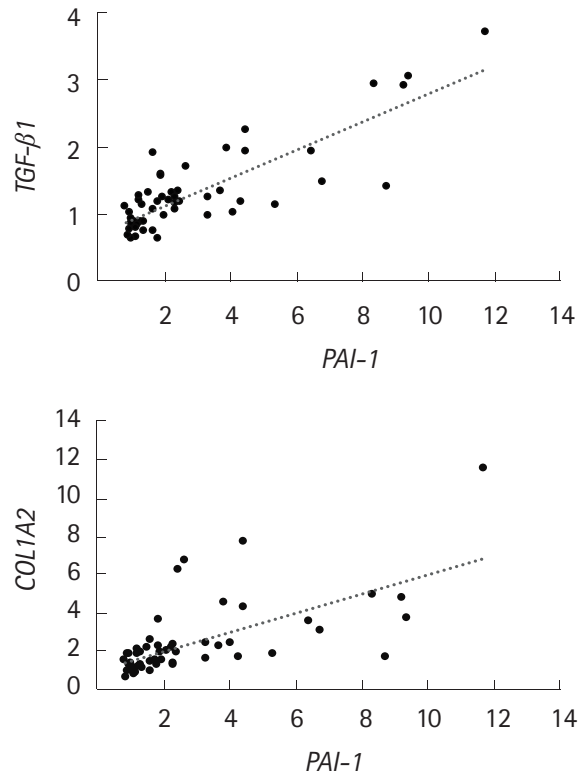

Fig. 1. The mRNA expression of PAl-1 is elevated in the terminal ileum of CD patients. The mRNA expression of PAl-1 (PAI-1), Col1A1 (COL1A1), Col1A2 (COL1A2) and TGF- $\beta 1$ (TGF- $\beta$ 1) genes in the ileal tissue from control subjects $(n=11)$, patients with inactive $C D(n=16)$ and active $C D$ $(n=51)$. Data were derived from a Gene Expression Omnibus (GEO) dataset GSE75214. (A) The expression of PAl-1 (PAl-1) was significantly upregulated in the terminal ileal mucosa of active CD patients compared with that of normal controls or inactive CD patients. (B) COL1A1 and TGF- $\beta 1$ are positively correlated. Correlation coefficient between PAl-1 and Col1A1 or Col1A2 are 0.73 or 0.75 . $P$-value by Kruskal-Wallis test. Correlation of 2 values using Pearson correlation coefficient. ${ }^{a} P<0.001$. PAI-1, plasminogen activator inhibitor- $1 ;$ TFG- $\beta$, transforming growth factor $\beta$. 
A

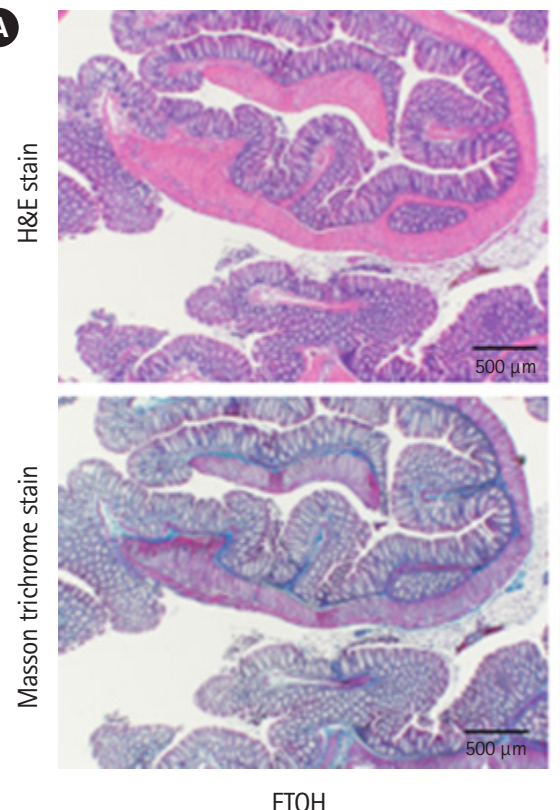

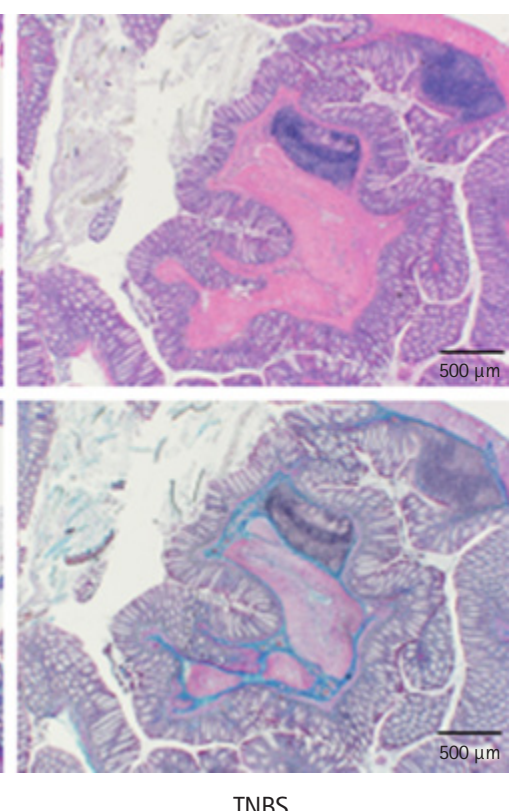

B

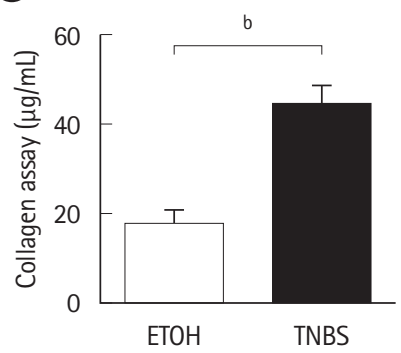

C

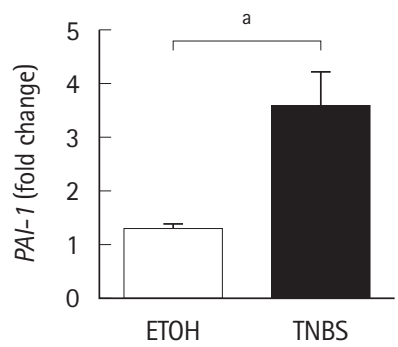

Fig. 2. TNBS-induced fibrotic model demonstrated the enhancement of plasminogen activator inhibitor-1 (Pai-1) expression. BALB/c mice were intrarectal injected with 2\% TNBS. (A) Representative histological examples of each group (H\&E and Masson trichrome staining). (B) Collagen deposition was determined by the Sircol collagen assay. (C) The mRNA expression of murine Pai- 1 in the colon samples was assessed by realtime PCR. Expression of the Pai-1 gene was normalized against that of the Gapdh gene in the same RNA preparation (5 ETOH, 10 TNBS). Bars represent the median. $P$-value by Mann-Whitney U-test. ${ }^{a} P<0.05 ;{ }^{b} P<0.01$. TNBS, 2,4,6-trinitrobenzene sulfonic acid; ETOH, ethanol.

compared with the control mice (Fig. 2C). The results suggested that repeated intrarectal injection of TNBS-induced intestinal fibrosis along with local high expression of Pai-1, which was similar to the findings in patients with $\mathrm{CD}$.

\section{PAI-1 Inhibitor Suppresses Intestinal Fibrosis in TNBS-Induced Murine Colitis}

To assess the role of PAI- 1 in TNBS-elicited intestinal fibrosis and to improve outcomes, we attempted to inhibit the signal by administering a PAI-1 inhibitor TM5275 after the collagen had accumulated ( 5 weeks later from the first treatment of TNBS). ${ }^{7,12}$ TM5275 is originally developed by ourselves and prevents PAI-1/tPA complex formation, thereby preserving active tPA. ${ }^{12,19}$ Histological analysis revealed that TM5275 administration decreased TNBS-induced collagen deposition only in the $50 \mathrm{mg} / \mathrm{kg}$ group, compared with that in the CMCadministered control group (Fig. 3A). The effects of TM5275 on the macroscopic and microscopic score of colitis were clearly observed in the thickness, strictures and dilation of colon (Table 2) and the depth of lesion, the degree of fibrosis (Table 3), which is also shown as the score in Fig. 3B and C. This indicated that TM5275 attenuated the TNBS-induced colonic fibrosis. Consistently, tissue collagen content was signifi- cantly reduced in the $50 \mathrm{mg} / \mathrm{kg}$ TM5275 group but not in the $15 \mathrm{mg} / \mathrm{kg}$ group (Fig. 3D). These results suggested that the PAI1 induction promoted collagen deposition and exacerbated colorectal fibrosis. In contrast, neither the loss of body weight nor the shortening of the gut was attenuated by the administration of TM5275 (Fig. 3E and F). These could be realized that the blockade of PAI-1 does not affect the chronic inflammation in the gut. PAI-1 reportedly promotes ECM deposition in fibrotic tissue by inhibiting MMP-9 production, and PAI-1 inhibition increases MMP-9 production. ${ }^{10,20,21}$ Consistent with these reports, we detected the MMP-9 upregulation in the 50 $\mathrm{mg} / \mathrm{kg}$ TM5275 group (Fig. 3G). The results indicated that targeting PAI-1 inhibition reduced collagen deposition via actual MMP-9 induction and that PAI-1 inhibitor could be useful in the treatment of intestinal fibrosis in patients with CD (Fig. 4).

\section{DISCUSSION}

Although the antifibrinolytic PAI-1 functions have received most of the attention in research on tissue fibrosis, ${ }^{22}$ more recent report has demonstrated a correlation between enhanced PAI-1 expression and inflammation in IBD patients and murine acute colitis model. ${ }^{11}$ In the present study, we ob- 
A
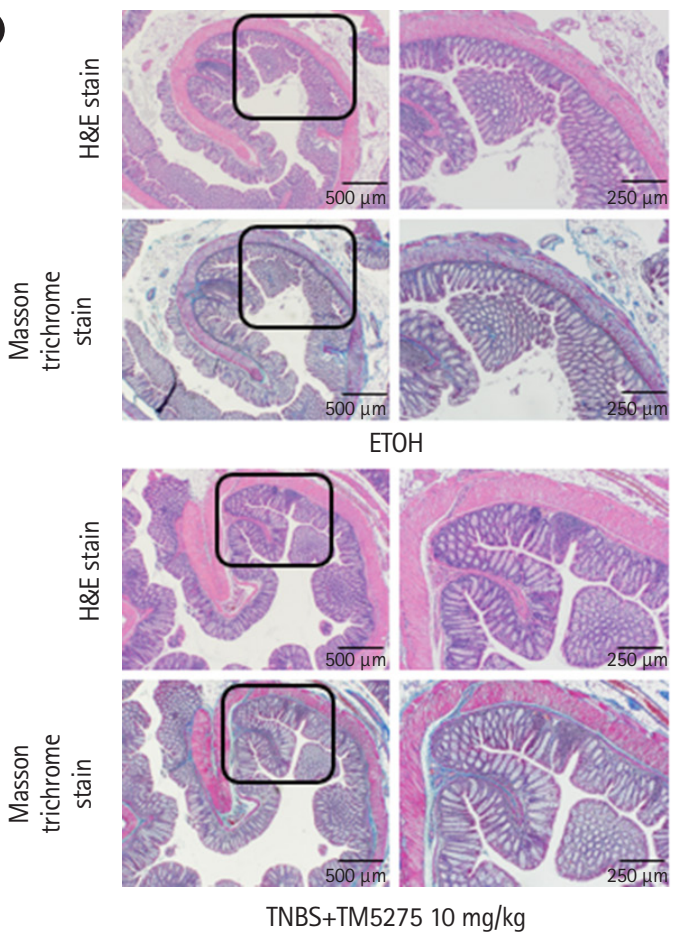

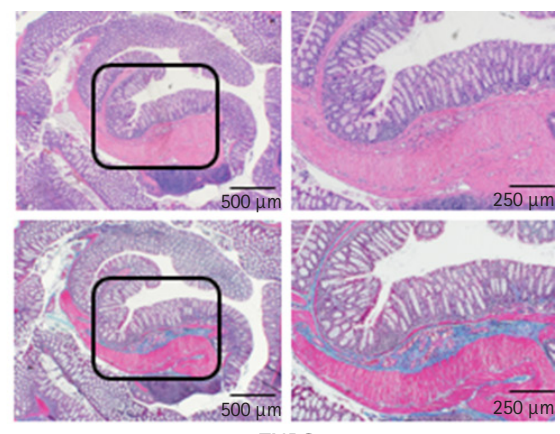

TNBS
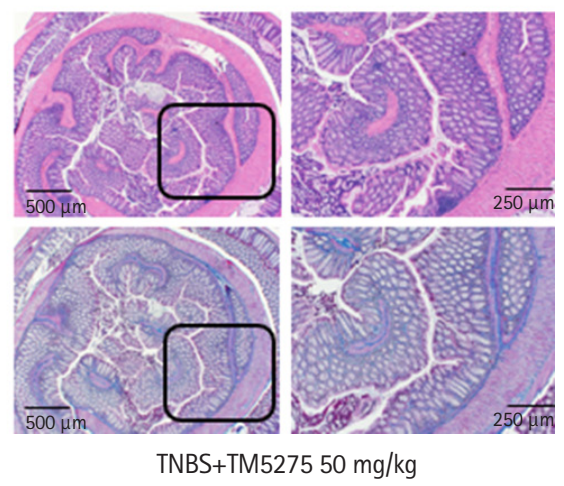

B

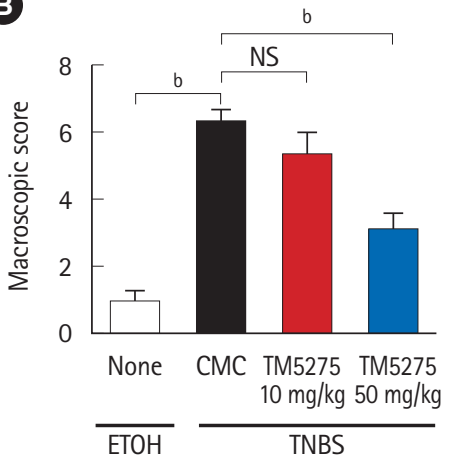

E

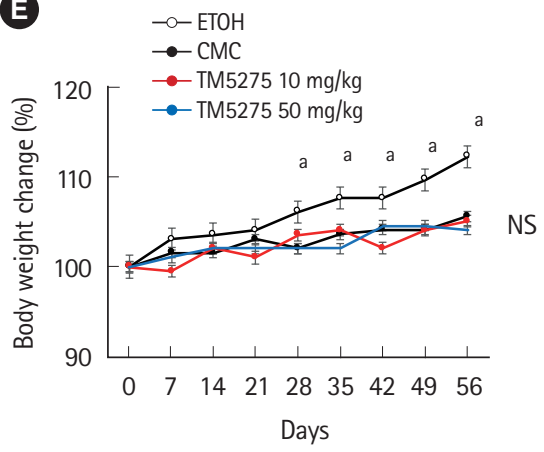

C

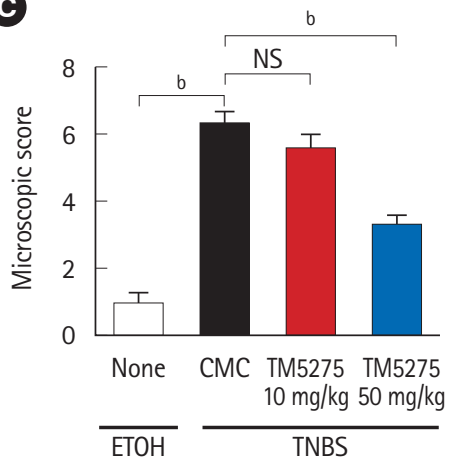

$\boldsymbol{F}$

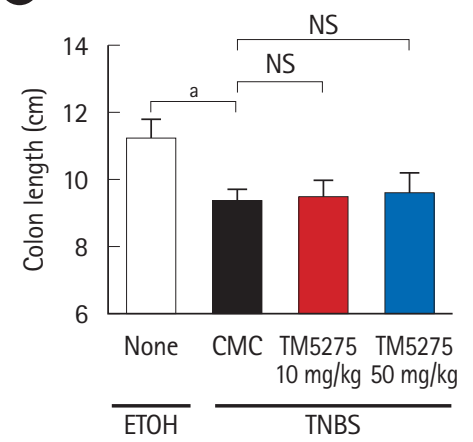

D

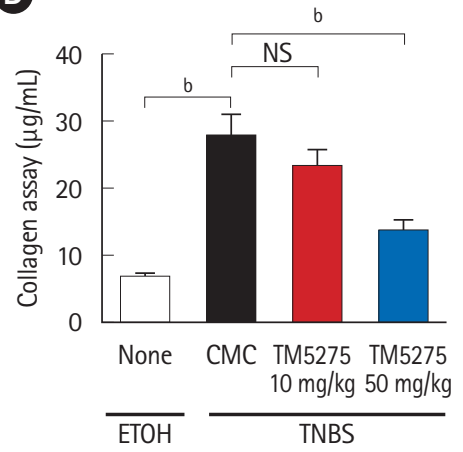

G

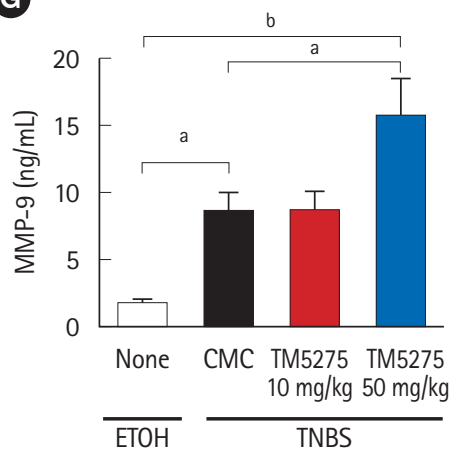

Fig. 3. PAl-1 inhibitor (TM5275) suppressed intestinal fibrosis in TNBS-induced murine colitis. (A) Representative histological examples of each group (H\&E and Masson trichrome staining). ( $B, C$ ) Analysis of macroscopic score and microscopic score. (D) Collagen deposition was determined by the Sircol collagen assay. (E) Body weight changes of each group. (F) Colon length of each group. (G) Local production of MMP-9 in the colonic mucosa of chronic colitis by ELISA. Bars represent the median. $P$-value by two-way ANOVA with Bonferroni post-hoc test. ${ }^{a} P<0.05$; ${ }^{b} P<0.001$. PAI-1, plasminogen activator inhibitor-1; TNBS, 2,4,6-trinitrobenzene sulfonic acid; MMP-9, matrix metalloproteinase 9; ETOH, ethanol; $C M C$, carboxymethyl cellulose. 
Table 2. Macroscopic Score of Colonic Fibrosis

\begin{tabular}{lcccccc}
\hline \multirow{2}{*}{ Group } & \multicolumn{5}{c}{ Macroscopic score of colonic fibrosis } \\
\cline { 2 - 7 } & Adhesion & Thickness & Strictures & Dilation & Mucosal edema & Total macroscopic score \\
\hline Ethanol $(n=10)$ & $0.20 \pm 0.42$ & $0.10 \pm 0.32$ & 0 & $0.20 \pm 0.42$ & $0.50 \pm 0.42$ & $1.00 \pm 0.67$ \\
CMC $(n=10)$ & $0.90 \pm 0.31$ & $1.80 \pm 0.42$ & $1.80 \pm 0.47$ & $0.80 \pm 0.42$ & $0.80 \pm 0.42$ & $6.10 \pm 0.87$ \\
TM5275 $10 \mathrm{mg} / \mathrm{kg}(n=9)$ & $0.74 \pm 0.44$ & $1.38 \pm 0.72$ & $1.65 \pm 0.44$ & $0.74 \pm 0.50$ & $0.74 \pm 0.44$ & $5.40 \pm 0.60$ \\
TM5275 $50 \mathrm{mg} / \mathrm{kg}(\mathrm{n}=10)$ & $0.80 \pm 0.42$ & $0.70 \pm 0.67^{\mathrm{a}, \mathrm{c}}$ & $0.80 \pm 0.63^{\mathrm{a}, \mathrm{c}}$ & $0.30 \pm 0.48^{\mathrm{a}, \mathrm{b}}$ & $0.50 \pm 0.53^{\mathrm{a}, \mathrm{b}}$ & $3.10 \pm 0.73^{\mathrm{a}, \mathrm{c}}$ \\
\hline
\end{tabular}

Values are presented as mean \pm SD.

${ }^{a}$ Statistical analyses were performed using two-way ANOVA with Bonferroni post-hoc test, which were compared with those of carboxymethyl cellulose (CMC) group.

${ }^{6} P<0.05$.

${ }^{c} P<0.001$.

Table 3. Microscopic Score of Colonic Fibrosis

\begin{tabular}{lccccc}
\hline \multirow{2}{*}{ Group } & \multicolumn{5}{c}{ Microscopic score of colonic fibrosis $^{\mathrm{a}}$} \\
\cline { 2 - 6 } & Ulcerations & Degree of inflammation & Depth of lesions & Degree of fibrosis & Total microscopic score \\
\hline Ethanol $(n=10)$ & $0.10 \pm 0.32$ & $0.20 \pm 0.42$ & $0.20 \pm 0.42$ & $0.30 \pm 0.48$ & $0.80 \pm 0.63$ \\
CMC $(n=10)$ & $0.90 \pm 0.31$ & $1.60 \pm 0.51$ & $1.80 \pm 0.48$ & $1.90 \pm 0.31$ & $6.20 \pm 0.63$ \\
TM5275 $10 \mathrm{mg} / \mathrm{kg}(n=9)$ & $0.83 \pm 0.63$ & $1.46 \pm 0.52$ & $1.56 \pm 0.51$ & $1.56 \pm 0.50$ & $5.30 \pm 0.62$ \\
TM5275 $50 \mathrm{mg} / \mathrm{kg}(n=10)$ & $0.70 \pm 0.48$ & $1.30 \pm 0.67$ & $0.90 \pm 0.56^{b}$ & $0.40 \pm 0.51^{b}$ & $3.30 \pm 0.67^{b}$ \\
\hline
\end{tabular}

Values are presented as mean \pm SD.

a Statistical analyses were performed using two-way ANOVA with Bonferroni post-hoc test, which were compared with those of carboxymethyl cellulose (CMC) group.

${ }^{b} P<0.001$.
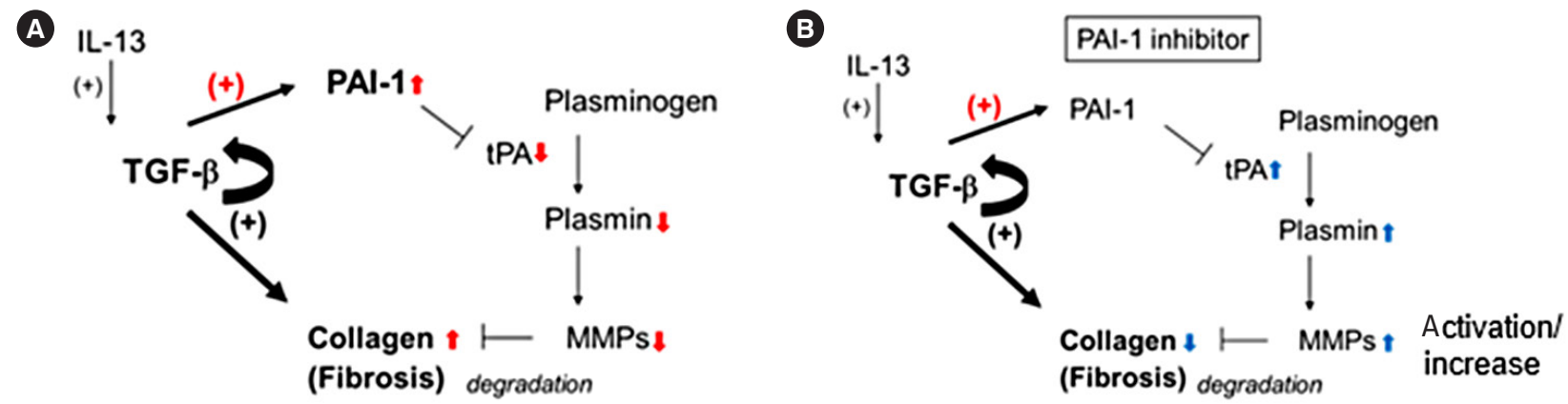

Fig. 4. The anti-fibrotic mechanism of plasminogen activator inhibitor-1 (PAI- 1 ) inhibitor. (A) Induced transforming growth factor $\beta$ (TGF- $\beta$ ) and its autocrine signaling (straight and semicircular arrows with + ) promote the deposition of collagen during colonic fibrosis in mice as shown in previous report. ${ }^{7}$ Simultaneously, it induces PAI-1 (arrow with red + ), which inactivates tissue plasminogen activator (tPA)/plasmin axis, and reduces the total amount and active matrix metalloproteinase 9 (MMP-9) (downward red arrows), resulting in the accumulation of collagen (upward red arrows). (B) Conversely, PAl- 1 inhibitor activates tPA/plasmin axis, up-regulates MMP-9 (upward blue arrows) and causes the degradation of collagen (downward blue arrows).

served PAI- 1 overexpression at active lesions in both patients with $\mathrm{CD}$ and mice with the chronic colitis; this overexpression seems to exacerbate intestinal fibrosis and PAI-1 inhibitor is a potential drug candidate for targeted therapy. As PAI-1 inhibition induced MMP-9 activation in the TNBS colitis model, PAI- 1-mediated MMP-9 activity inhibition could be a key mechanism via which ECM is deposited in the lesions. ${ }^{20}$

A large number of studies have focused on the molecular mechanisms responsible for the TGF- $\beta$-stimulated type-I collagen gene transcription and its pathological roles during the 
fibrotic process. ${ }^{23-25}$ Because TGF- $\beta$ is a potent anti-inflammatory cytokine, its signal blockade seems to exacerbate inflammation. TGF- $\beta 1$-deficient mice develop severe multi-organ inflammation, ${ }^{26,27}$ and targeted deletions of Smad 2 and 4 are associated with early death in mice, ${ }^{28,29}$ highlighting the need for caution in applying anti-TGF- $\beta$ strategies as anti-fibrotic therapies. In addition to the direct induction of collagen, we focused on TGF- $\beta$ attenuating the fibrinolytic system via PAI-1 expression as its downstream target. Therefore, targeting PAI-1 inhibition could facilitate intestinal fibrosis treatment with minimal or no side effects.

MMP-9 has previously been identified as a key marker of inflammation in both UC and $\mathrm{CD}^{30,31}$ and as effective in the development of murine colitis. ${ }^{32}$ Similarly, plasmin inhibition reportedly protects against colitis in mice by suppressing MMP$9{ }^{21}$ However, a recent report argued against its significance because MMP-9 inhibition does not attenuate murine colitis. ${ }^{33}$ In addition, recent clinical trials with MMP-9 antagonist have not demonstrated significant efficacy in patients with moderate-to-severe UC or $\mathrm{CD} .^{34}$ Such results obscure the significance of MMP-9 for the regulation of inflammation. Contrary to their validation methods, which focus on the acute phase of colitis, in the present study, we focus on the significance of PAI-1 for intestinal fibrosis in the chronic phase of colitis, where MMP-9 seems to be effective in the degradation of fibrotic deposition. Because MMP-9 plays multiple roles at different stages of IBD development, anti-MMP-9 therapy should be developed with caution. Previous report indicated that tPA-mediated active plasmin increases the total amount of MMP-9 in the gut with acute inflammation. ${ }^{21}$ Although its precise mechanism has not been fully understood yet, there may be another pathway to induce MMP-9 by active plasmin. This is quite consistent with our finding that the blockade of PAI-1 activity, probably activates $\mathrm{tPA}$ /plasmin axis, resulting in the upregulation of total MMP-9 (Fig. 4). Alternatively, PAI-1-mediated fibrinolytic system could regulate other protease(s) to degrade ECM deposition; however, this aspect requires further investigation.

PAI-1 inhibitors that we developed are currently under evaluation in phase II clinical trials for the treatment of chronic myeloid leukemia with Imatinib in Japan. If the drugs exhibit a potential to attenuate intestinal fibrosis in IBD, it could facilitate further indication and clinical research. Because only few anti-fibrotic drugs are available for the treatment of various fibrotic lesions in multiple organs, more novel approaches are required to facilitate processes that could lead to the development of effective therapies.

\section{FINANCIAL SUPPORT}

This study was supported by the Ministry of Education, Culture, Sports, Science and Technology (MEXT; 17H05802 for Hozumi K); Japan Society for the Promotion of Science (JSPS; 19K017413 for Imai J); a MEXT-Supported Program for the Strategic Research Foundation at Private University (Tokai University) for Imai Y.

\section{CONFLICT OF INTEREST}

No potential conflict of interest relevant to this article was reported.

\section{AUTHOR CONTRIBUTION}

Conceptualization: Hozumi K, Imai J, Yahata T. Funding acquisition: Hozumi K, Imai J. Methodology: Hozumi K, Imai J, Yahata T, Ichikawa H, Ibrahim AA, Yazawa M, Sumiyoshi H. Supervision: Ichikawa H, Inagaki Y, Matsushima M, Suzuki T, Mine T, Ando K, Miyata T. Writing original draft: Imai J, Hozumi K. All authors read and approved the final version of the manuscript.

\section{ORCID}

Imai J https://orcid.org/0000-0001-5248-4543

Yazawa M https://orcid.org/0000-0003-2382-9033

Sumiyoshi H https://orcid.org/0000-0003-0747-579X

Inagaki Y https://orcid.org/0000-0001-6640-7688

Hozumi K https://orcid.org/0000-0002-7685-6927

\section{ACKNOWLEDGEMENTS}

Authors would like to thank the members of the Support Center for Medical Research and Education, Tokai University, for their experimental support.

\section{REFERENCES}

1. Cosnes J, Gower-Rousseau C, Seksik P, Cortot A. Epidemiology and natural history of inflammatory bowel diseases. Gastroenterology 2011;140:1785-1794.

2. Latella G, Sferra R, Speca S, Vetuschi A, Gaudio E. Can we prevent, reduce or reverse intestinal fibrosis in IBD? Eur Rev Med Pharmacol Sci 2013;17:1283-1304. 
3. Speca S, Giusti I, Rieder F, Latella G. Cellular and molecular mechanisms of intestinal fibrosis. World J Gastroenterol 2012; 18:3635-3661.

4. Rieder F, Fiocchi C, Rogler G. Mechanisms, management, and treatment of fibrosis in patients with inflammatory bowel diseases. Gastroenterology 2017;152:340-350.

5. Bettenworth D, Rieder F. Medical therapy of stricturing Crohn's disease: what the gut can learn from other organs: a systematic review. Fibrogenesis Tissue Repair 2014;7:5.

6. Verrecchia F, Mauviel A. Transforming growth factor-beta and fibrosis. World J Gastroenterol 2007;13:3056-3062.

7. Imai J, Hozumi K, Sumiyoshi H, et al. Anti-fibrotic effects of a novel small compound on the regulation of cytokine production in a mouse model of colorectal fibrosis. Biochem Biophys Res Commun 2015;468:554-560.

8. Higashi K, Tomigahara Y, Shiraki H, et al. A novel small compound that promotes nuclear translocation of YB-1 ameliorates experimental hepatic fibrosis in mice. J Biol Chem 2011; 286:4485-4492

9. Samarakoon R, Higgins PJ. Integration of non-SMAD and SMAD signaling in TGF-betal-induced plasminogen activator inhibitor type-1 gene expression in vascular smooth muscle cells. Thromb Haemost 2008;100:976-983.

10. Ghosh AK, Vaughan DE. PAI-1 in tissue fibrosis. J Cell Physiol 2012;227:493-507.

11. Kaiko GE, Chen F, Lai CW, et al. PAI-1 augments mucosal damage in colitis. Sci Transl Med 2019;11:eaat0852.

12. Ibrahim AA, Yahata T, Onizuka M, et al. Inhibition of plasminogen activator inhibitor type-1 activity enhances rapid and sustainable hematopoietic regeneration. Stem Cells 2014;32: 946-958.

13. Latella G, Vetuschi A, Sferra R, et al. Smad3 loss confers resistance to the development of trinitrobenzene sulfonic acid-induced colorectal fibrosis. Eur J Clin Invest 2009;39:145-156.

14. Lawrance IC, Wu F, Leite AZ, et al. A murine model of chronic inflammation-induced intestinal fibrosis down-regulated by antisense NF-kappa B. Gastroenterology 2003;125:1750-1761.

15. Fichtner-Feigl S, Fuss IJ, Young CA, et al. Induction of IL-13 triggers TGF-betal-dependent tissue fibrosis in chronic 2,4,6-trinitrobenzene sulfonic acid colitis. J Immunol 2007;178:58595870.

16. Higashi K, Inagaki Y, Fujimori K, Nakao A, Kaneko H, Nakatsuka I. Interferon-gamma interferes with transforming growth factor-beta signaling through direct interaction of YB-1 with Smad3. J Biol Chem 2003;278:43470-43479.

17. Gasche C, Scholmerich J, Brynskov J, et al. A simple classifica- tion of Crohn's disease: report of the Working Party for the World Congresses of Gastroenterology, Vienna 1998. Inflamm Bowel Dis 2000;6:8-15.

18. Vancamelbeke M, Vanuytsel T, Farré R, et al. Genetic and transcriptomic bases of intestinal epithelial barrier dysfunction in inflammatory bowel disease. Inflamm Bowel Dis 2017;23: 1718-1729.

19. Yahata T, Ibrahim AA, Muguruma Y, et al. TGF-beta-induced intracellular PAI-1 is responsible for retaining hematopoietic stem cells in the niche. Blood 2017;130:2283-2294.

20. Oh CK, Ariue B, Alban RF, Shaw B, Cho SH. PAI-1 promotes extracellular matrix deposition in the airways of a murine asthma model. Biochem Biophys Res Commun 2002;294: 1155-1160.

21. Munakata S, Tashiro Y, Nishida C, et al. Inhibition of plasmin protects against colitis in mice by suppressing matrix metalloproteinase 9-mediated cytokine release from myeloid cells. Gastroenterology 2015;148:565-578.

22. Pincha N, Hajam EY, Badarinath K, et al. PAIl mediates fibroblast-mast cell interactions in skin fibrosis. J Clin Invest 2018; 128:1807-1819.

23. Rieder F, Bettenworth D, Imai J, Inagaki Y. Intestinal fibrosis and liver fibrosis: consequences of chronic inflammation or independent pathophysiology? Inflamm Intest Dis 2016;1:4149.

24. Inagaki Y, Okazaki I. Emerging insights into transforming growth factor beta Smad signal in hepatic fibrogenesis. Gut 2007;56:284-292.

25. Li C, Iness A, Yoon J, et al. Noncanonical STAT3 activation regulates excess TGF-betal and collagen I expression in muscle of stricturing Crohn's disease. J Immunol 2015;194:3422-3431.

26. Diebold RJ, Eis MJ, Yin M, et al. Early-onset multifocal inflammation in the transforming growth factor beta 1-null mouse is lymphocyte mediated. Proc Natl Acad Sci U S A 1995;92: 12215-12219.

27. Kulkarni AB, Ward JM, Yaswen L, et al. Transforming growth factor-beta 1 null mice: an animal model for inflammatory disorders. Am J Pathol 1995;146:264-275.

28. Nomura M, Li E. Smad2 role in mesoderm formation, leftright patterning and craniofacial development. Nature 1998; 393:786-790

29. Yang X, Li C, Xu X, Deng C. The tumor suppressor SMAD4/ DPC4 is essential for epiblast proliferation and mesoderm induction in mice. Proc Natl Acad Sci U S A 1998;95:3667-3672.

30. de Bruyn M, Arijs I, Wollants WJ, et al. Neutrophil gelatinase B-associated lipocalin and matrix metalloproteinase-9 com- 
plex as a surrogate serum marker of mucosal healing in ulcerative colitis. Inflamm Bowel Dis 2014;20:1198-1207.

31. de Bruyn M, Vandooren J, Ugarte-Berzal E, Arijs I, Vermeire S, Opdenakker G. The molecular biology of matrix metalloproteinases and tissue inhibitors of metalloproteinases in inflammatory bowel diseases. Crit Rev Biochem Mol Biol 2016;51: 295-358.

32. Castaneda FE, Walia B, Vijay-Kumar M, et al. Targeted deletion of metalloproteinase 9 attenuates experimental colitis in mice: central role of epithelial-derived MMP. Gastroenterology 2005;129:1991-2008.

33. De Bruyn M, Breynaert C, Arijs I, et al. Inhibition of gelatinase B/MMP-9 does not attenuate colitis in murine models of inflammatory bowel disease. Nat Commun 2017;8:15384.

34. De Bruyn M, Ferrante M. Failure of MMP-9 antagonists in IBD: demonstrating the importance of molecular biology and well-controlled preclinical studies. J Crohns Colitis 2018;12: 1011-1013. 